\title{
PRE-MARKET CLINICAL EVALUATIONS OF INNOVATIVE HIGH-RISK MEDICAL DEVICES IN EUROPE
}

Frank Hulstaert

Belgian Health Care Knowledge Center (KCE)

Email: frank.hulstaer@@kce.fgov.be

Mattias Neyt, Imgard Vinck, Sabine Stordeur

Belgian Health Care Knowledge Center (KCE)

Mirjana Huic

Agency for Quality and Accreditation in Health

Stefan Saverland

Institute for Quality and Efficiency in Healthcare (IQWiG)

Marja R. Kuïpers, Payam Abrishami

Dutch Health Care Insurance Board (CVZ)
Hindrik Vondeling

Centre for Applied Health Services Research and Technology Assessment (CAST)

Bruno Flamion

Medical Faculty, University of Namur

Silvio Garattini

Mario Negri Research Institute, Institute for Pharmacological Research

Mira Pavlovic

Haute Autorité de Santé

Hans van Brabandt

Belgian Health Care Knowledge Center (KCE)

Objectives: High-quality clinical evidence is most often lacking when novel high-risk devices enter the European market. At the same time, a randomized controlled trial (RCT) is often initiated as a requirement for obtaining market access in the US. Should coverage in Europe be postponed until RCT data are available? We studied the premarket clinical evaluation of innovative high-risk medical devices in Europe compared with the US, and with medicines, where appropriate.

Methods: The literature and regulatory documents were checked. Representatives from industry, Competent Authorities, Notified Bodies, Ethics Committees, and HTA agencies were consulted. We also discuss patient safety and the transparency of information.

Results: In contrast to the US, there is no requirement in Europe to demonstrate the clinical efficacy of high-risk devices in the premarket phase. Patients in Europe can thus have earlier access to a potentially lifesaving device, but at the risk of insufficiently documented efficacy and safety. Variations in the stringency of clinical reviews, both at the level of Notified Bodies and Competent Authorities, do not guarantee patient safety. We tried to document the design of premarket trials in Europe and number of patients exposed, but failed as this information is not made public. Furthermore, the Helsinki Declaration is not followed with respect to the registration and publication of premarket trials.

Conclusions: For innovative high-risk devices, new EU legislation should require the premarket demonstration of clinical efficacy and safety, using an RCT if possible, and a transparent clinical review, preferably centralized.

Keywords: Medical device, Device approval, Equipment and supplies, European Union, United States Food and Drug Administration

We thank Dirk Cuypers (Ministry of Health) for placing this topic on the agenda of the EU Member State Chief Medical Officers Meeting, Liège, Belgium, November 2010. We are grateful to Xavier De Cuyper and Vanessa Biname of the Federal Agency for Medicines and Health Products FAGG/AFMPS for their support in setting up the survey among the Notified Bodies. Our thanks go also to Marc Bogaert (Belgian Advisory Committee on Bioethics), Pete Wall (Isca Healthcare Research and Research Ethics (ommittee for Wales, UK), Jean-Marie Maloteaux (Ethics Committee, UCL University Hospital St-Luc, Brussels), and Kris Dierickx (Centre for Biomedical Ethics and Law, University of Leuven) for providing input on medical ethics and feedback from their Ethics Committees. We received helpful clarifications from representatives of the Competent Authorities, in particular Suzanne Ludgate (Medicines and Healthcare Products Regulatory Agency, UK) and Niall MacAleenan (lrish Medicines Board), as well as from William Sutton (Food and Drug Administration, Center for Devices and Radiological Health, Division for International Assistance). We are also grateful to Gert Bos (BSI Notified Body) and Trevor Lewis (Medical Device Consultancy) for sharing their points of view. The research was funded by the Belgian Health Care Knowledge Centre (KCE). This manuscript is based on an approved KCE research project that was initiated and designed by Frank Hulstaert and Mattias Neyt. Mattias Neyt, Sabine Stordeur, and Hans Van Brabandt contributed with practical examples of
Evidence of effectiveness is increasingly requested by payers to support coverage decisions, and also for medical devices (22). Health Technology Assessment (HTA) agencies are repeatedly confronted with a lack of high-level clinical evidence when assessing the value of innovative high-risk devices when they enter the market in Europe. At the same time, a

evaluations of innovative high-risk devices at KCE. Imgard Vinck contributed with research ethics and medico-legal aspects. Miriana Huic, Stefan Sauerland, Maria R. Kuijpers, Payam Abrishami, Hindrik Vondeling, Bruno Flamion, Silvio Garattini, and Mira Pavlovic were instrumental in improving earlier draft versions of the report during various stages of this project.

The online version of this article is published within an Open Access environment subject to the conditions of the Creative Commons Attribution-NonCommercial-ShareAlike licence $<$ hitp://creativecommons.org/licenses/by-nc-sa/2.5/>. The written permission of Cambridge University Press must be obtained for commercial re-use. 
randomized controlled trial ( $\mathrm{RCT})$ is often initiated as a requirement for obtaining market access in the United States. These RCTs are sometimes partly conducted in Europe with CE (Conformité Européenne) marked products $(9 ; 19)$. Similar observations were made in three rapid assessments by the Belgian Health Care Knowledge Centre (KCE), covering percutaneous aortic valves, endobronchial valves and interspinous devices $(26 ; 30 ; 31)$. Payers in Europe are placed in a difficult situation. Postponing the reimbursement decision until the RCT results are available is often met with mixed feelings by industry and clinicians. For the patient, the current system in Europe can mean earlier access to a potentially lifesaving device, but at the risk of insufficiently documented efficacy and safety. Furthermore, the cost of the device may be fully charged to the patient. This growing tension between early market introduction and the reimbursement of innovative high-risk devices in Europe led us to take a closer look at the premarket clinical evaluation of such devices.

Both in Europe and the United States, medical devices are categorized into different classes based on the risk they pose to patients, with class III devices representing the highest risk category. We define innovative high-risk devices in Europe as class III devices and implantable devices, that differ more than slightly from existing devices in the same indication with regard to design, expected effectiveness, or both. In addition, the use of a high-risk device (new or existing) in a new indication has also to be considered an innovation. In the United States, innovative high-risk devices (class III) typically undergo a premarket approval (PMA) process.

Contrary to what health professionals and users of high-risk medical devices may assume, the premarket clinical evaluation of high-risk medical devices in Europe is not comparable to the evaluation of pharmaceuticals in terms of safety and efficacy. Patient safety aspects of the European approach for high-risk devices have not been widely discussed in peer reviewed journals. During the finalization of our study, a policy report by the European Society of Cardiology was published (13), as well as a series of articles in the British Medical Journal $(4 ; 12 ; 14 ; 28 ; 32)$ that call for more transparency and a higher quality clinical evaluation of medical devices in Europe during both pre- and postmarketing.

The aim of this study was to review and discuss the premarket clinical evaluation of innovative high-risk medical devices in Europe and to compare it with evaluations in the United States. We also compare the evaluation of devices with the evaluation of medicines, where appropriate. In addition, we discuss issues regarding patient safety and the transparency of information. This report does not cover the preclinical evaluation or the manufacturing practices of devices. Furthermore, our conclusions should not be extrapolated to the vast number of lower risk devices outside the scope of this study. The full $\mathrm{KCE}$ report (number 158) on which this manuscript is based is available at www.kce.fgov.be.

\section{METHODS}

We consulted the peer reviewed literature using a Medline search. The yield of this search was very poor, at least for descriptions and critical reviews of the European system when compared with the United States.

We consulted experts of member organizations of EUnetHTA (a European network of HTA agencies), and we checked the grey literature and European and U.S. regulatory documents. Furthermore, representatives from Competent Authorities, the U.S. Food and Drug Administration (FDA), Ethics Committees, and the Medical Device Industry were consulted.

Three major Notified Bodies were requested by Belgian Competent Authorities to complete a simple questionnaire on the number of patients exposed to the innovative device preCE mark and on the type of trial design (randomized or not). This information (without identifying the device) was requested for cardiovascular implantable devices with or without an active substance and for other implantable devices with an active substance.

The list of study protocols reviewed by Belgian Ethics Committees in 2010 was searched for trials with high-risk medical devices by Professor Marc Bogaert, Belgian Advisory Committee on Bioethics. In addition, the Ethics Committees were requested to provide the regulatory status of the device under study.

\section{Clinical Development Phases}

The clinical development of medical interventions and technologies for a given indication is characterized by different phases. Exploratory studies, often generating a hypothesis, are followed by confirmatory studies to test the hypothesis (17). This phased approach is now the standard for the premarket clinical development of pharmaceuticals. Exploratory trials of high-risk devices are more complex compared with drug trials: it is not just the device itself that is studied but the complete system. This includes the most optimal procedures for handling the device and ways of shortening the learning curve. In addition, exploratory device trials often lead to minor modifications of the new device, a phenomenon that makes it even more difficult to determine when an innovation is ready for confirmatory studies.

Randomized control trials (RCTs) are the highest standard for documenting clinical safety and demonstrating any incremental efficacy, also for devices. For example, in the absence of well-controlled trials, it may be impossible to judge whether a 30-day mortality rate of 5 or 10 percent after the implantation of a percutaneous aortic valve should be considered high or low (30). High quality RCTs can also be conducted with devices, even if these trials involve no or only the partial blinding of patients, physicians, or outcome assessors (14). Experience at the FDA has shown that valid and realistic study designs are possible for the controlled evaluation of the efficacy and safety of medical devices. The FDA considers RCTs to be critical for 
Hulstaert et al.

Table 1. The sequence of clinical development and timing of the market introduction of novel invasive high-risk devices in Europe and the US

Theoretical sequence over time
Exploratory clinical trials
Confirmatory clinical
trials (RCTs)
Health technology assessment

\begin{abstract}
CE mark system in Europe
Market introduction based on single-arm trials

demonstrating safety and "performance"
\end{abstract}

FDA PMA process in the US

\author{
Market introduction based on RCTs \\ demonstrating safety and \\ "efficacy/effectiveness"
}
Requires safety and efficacy/effectiveness data
for the assessment, which may include an
evaluation of cost-effectiveness

statistical validity, and they are ethical if designed according to current U.S. Good Clinical Practice. Randomized controlled trials with sham (or placebo) surgery as a comparator for proving device efficacy may sometimes be considered unethical. It may be more appropriate to document efficacy versus the reference treatment (watchful waiting, another device, drug treatment, conventional surgery, etc.). To not expose too many patients unnecessarily to a high-risk intervention, the conduct of an RCT should not be delayed. This was recently illustrated for stents used to treat intracranial arterial stenosis, where the stents had been approved 6 years previously based on a humanitarian device exemption and on the registry data of only forty-five patients. After thousands of patients received the device, an RCT was stopped early because the 30-day rate of stroke or deaths was significantly higher than after medical treatment (3).

\section{Different Systems of Premarket Clinical Evaluation in Europe and the United States}

The premarket regulations of the FDA and Europe remain very different despite the creation of a Global Harmonization Task Force (GHTF) for device regulations in 1992 (www.ghtf.org) (18).

The European system is based on the demonstration of safety and performance, whereas the U.S. system requires the premarket demonstration of safety and efficacy/effectiveness (Table 1). This leads to entirely different clinical trials. For the demonstration of device performance, an RCT is neither necessary nor appropriate, whereas it is essential for the demonstration of clinical safety and efficacy in a controlled way. The level of study evidence required in Europe is also much less specified compared with the FDA requirements for a PMA application. The net result is an earlier market introduction in Europe compared with the United States.

\section{Demonstration of Device Performance in Europe}

In Europe, novel high-risk medical devices do not undergo a premarket review by Member States or European Authorities.
Instead, they have to go through a system of CE (Conformite Européenne) marking, which is mainly based on the 1993 Medical Device Directive, which was amended in 2007.

Conformité Européenne marking for high-risk novel medical devices is applied for by the manufacturer and the conformity assessment is checked by a Notified Body. Notified Bodies are for-profit organizations certified by a Member State Competent Authority. The latter vary between Member States. In some cases, national medicines agencies are endowed with the overview of medical devices; in other cases, specific agencies have been set up and, finally, some Member States do not have any specific pathway for the approval of medical devices, relying purely on Notified Bodies.

This premarket evaluation of a device by a Notified Body is performed only once for the entire European market. It includes the assessment and verification of the clinical evaluation, defined as the assessment and analysis of clinical data pertaining to a medical device to verify the clinical safety and performance of the device when used as intended by the manufacturer. It is very important to note that an evaluation of clinical efficacy is not part of the premarketing evaluation in Europe.

Member States can invoke a safeguard clause if evidence of a major public health concern is identified. In the case of devices containing a human blood derivative, the Notified Body can only decide positively if the European Medicines Agency's scientific opinion is favorable (18). However, for drug-device combination products, the Notified Body can overrule the opinion of the Medicines Agency.

There are approximately eighty Notified Bodies currently designated for a total of thirty-one countries in the European Economic Area (EEA). Some Notified Bodies only have two or three staff (4). Notified Bodies are not on a level playing field in terms of their criteria for approval; companies can work with different Notified Bodies for different devices and Competent Authorities are aware that companies could select a Notified Body that is likely to be less stringent in the assessment of a particular device $(4 ; 29)$. The Notified Body Operating Group 
(http://www.nbog.eu/) is working on this issue and the ongoing revision of the device directives is expected to provide a solution (5).

Directive 2007/47/EC, effective from March 2010, states that, in the case of implantable devices and class III devices, clinical investigations shall be performed unless reliance on existing clinical data is duly justified (27). Since May 2011, all clinical investigations (protocol title and primary objective only) are entered into a nonpublic database (Eudamed) by the Competent Authority where the trial is notified first (8); however, the trial authorization procedure differs significantly by Member State. Some Competent Authorities actively review the documentation submitted, including the trial design. Other Member States, however, have opted for the passive permission route (no review).

The December 2010 MEDDEV 2.7/4 guidelines (7) do not provide any specific requirements regarding the depth and extent of premarketing clinical evaluations of high-risk devices. The RCT design is not specifically endorsed.

\section{Premarket Clinical Trials in Europe Are not Made Public}

Based on grey literature (29) and informal contacts within Competent Authorities, it seems that the number of patients evaluated in premarket trials in Europe varies by the indication but is typically less than 100 patients. These studies are often referred to as single-arm "feasibility" trials or "performance" trials. Only for drug-eluting stents is the minimum number of patients exposed in the premarket phase more or less in the public domain: based on the potential safety issues that were identified, the number of patients studied increased over time from 50 to 300 (no RCT required).

Different initiatives to obtain more information on premarket clinical data did not succeed. Belgian Competent Authorities received no answer from three large Notified Bodies asked to provide the numbers of patients exposed and the trial design used for the novel high-risk devices they had approved. In the UK, researchers failed to obtain premarket clinical data from the manufacturers and six Notified Bodies for 192 recalled devices $(15 ; 28)$. Only four companies (2 percent) provided any clinical data. In the Netherlands, Competent Authorities encountered difficulties in obtaining technical documentation of class III devices from industry (coronary stents, total hip implants, and silver-containing wound dressings) (23). It is of interest to note that even Competent Authorities have to rely on a Google internet search to identify the class III devices being marketed as there was (and still is) no comprehensive list or database. Major shortcomings were identified in the documentation received, in particular concerning the clinical evaluation of the device (23). Confidentiality seems to overrule transparency in Europe much more than in the United States (4).

Based on a large subset of protocols that were reviewed in 2010, representatives of Belgian Ethics Committees reported that nearly all trials with high-risk devices in Belgium are con- ducted after the CE mark has been obtained, and that the identified pre-CE mark trials were not randomized.

\section{Premarket Clinical Review in the United States}

In the United States, premarket device review procedures were introduced in 1976. The evaluations are performed by the FDA, mainly by its Center for Devices and Radiological Health $(\mathrm{CDRH})$. For many devices entering the U.S. market the "premarket notification" route, the $510(\mathrm{k})$, applies. Innovative highrisk medical devices, however, are, in principle, subject to a more stringent procedure called the "premarket approval" (PMA) procedure, which typically requires the conduct of a randomized controlled trial (16).

Under the PMA process each manufacturer must independently demonstrate "reasonable assurance of the safety and effectiveness" of its device for its intended use. To start research trials in the United States to be included in a PMA, the sponsor first needs to obtain an Investigational Device Exemption (IDE) approval from the FDA (24). For diseases or conditions affecting small patient populations (under 4,000 patients in the United States), the FDA, since 1996, has allowed Humanitarian Device Exemption (HDE) applications, which are similar in both form and content to a PMA application, but are exempt from the effectiveness requirements of a PMA.

Nevertheless, PMA applications have been criticized as they often contain only a single study or they lack adequate strength or coverage of specific study populations $(6 ; 16 ; 20)$. Recent high-profile cases involving potentially dangerous defects in widely used cardiovascular devices have increased concerns about the adequacy of premarket trials and postmarket surveillance for establishing the safety of these devices. Furthermore, the $510(\mathrm{k})$ process has also been the subject of debate. A 2011 report by the U.S. Institute of Medicine (www.iom.edu) called for a new regulatory framework using a risk-based approach to replace the $510(\mathrm{k})$ process $(2)$.

\section{Other Effects of the Difference in Systems}

Another poorly documented effect that might be linked to the difference in regulatory systems is that only one in five of the 8,500 medical device companies in Europe (probably fewer if one excludes the companies belonging to a U.S. group) has approached the U.S. market (1). In addition, more devices of a particular type are often marketed in Europe compared with the United States; for example, 28 drug-eluting stents are CE marked whereas only 5 have obtained FDA approval. Unfortunately, final negative decisions on PMA dossiers are not made public on the FDA Web site. Therefore, the possibility that devices continue to be marketed in Europe even though they failed to demonstrate efficacy and safety in the RCT required in the context of a PMA in the United States cannot be excluded. This is illustrated by the negative FDA panel vote for an endobronchial valve system in 2008 (10). Several similar 
Table 2. Transparency of trials and their results for devices and pharmaceuticals in Europe and the US

\begin{tabular}{|c|c|c|}
\hline & Medical Devices & Medicines \\
\hline Europe & $\begin{array}{l}\text { Trial registry (Eudamed) not public* } \\
\text { Trial results not public* }\end{array}$ & Public trial registry (EudraCT) and results (EPAR) \\
\hline US & Public trial registry and results (clinicaltrials.gov) & Public trial registry and results (clinicaltrials.gov) \\
\hline
\end{tabular}

examples are given in a recent study by Cohen and Billingsley (4).

\section{Premarket Demonstration of Clinical Efficacy is Needed in Europe}

The discrepancy between the European and U.S. situations highlights the need for a significant change in legislation on this side of the Atlantic. The FDA believes that RCTs are critical for statistical validity. In a recent report of the World Health Organization, regulatory agencies were called upon to ensure that, whenever possible, high quality randomized trials are completed before granting marketing approval of high-risk medical devices (33).

Despite the increase in clinical trial activity induced by the EU Directive 2007/47/EC, the remaining variations in the stringency of clinical reviews both at the level of Notified Bodies and Competent Authorities do not guarantee patient safety in a uniform way for EU citizens. Therefore, for innovative highrisk devices, the future EU Device Directive should go beyond requiring safety and "device performance" data only to also requiring premarket data that demonstrate "clinical safety and efficacy". It should also be noted that for devices where only slight modifications were made to an existing device, a shortened development cycle may or may not be appropriate. This needs to be evaluated case by case.

Members of Ethics Committees and Competent Authorities are often confronted by a lack of clinical research expertise at small device companies. In addition, Notified Bodies may also lack the expertise to interpret clinical data and clinical safety signals (28). In this regard, the European Society of Cardiology has recommended that independent expert physicians should be involved in reviewing the decisions of new class III devices (13).

Initiatives to develop international guidance documents for premarket clinical trials by the type of high-risk device need to be stimulated (12). Such guidance documents have been proven to be of value for the more standardized, high quality clinical development of medicinal products. Not only professional societies (13) but, ideally, HTA agencies should also be involved in this activity, for example by proposing study endpoints, which will be needed for cost-effectiveness evaluations. Such documents should also further the standardization of evaluations across Notified Bodies and Competent Authorities. Industry could benefit if a positive reimbursement can be better planned and if potential liability risks of entering the market too early can be reduced.

Instead of trying to streamline a very fragmented system of Notified Bodies and Competent Authorities, a more straightforward way of achieving the goals discussed above could be to centralize expertise at the European level, as was done for advanced therapy medicinal products, and as suggested by the European Society of Cardiology (13). The transfer of evaluation and approval to a centralized body may, however, be more urgent for innovative high-risk devices than for other lower risk devices.

Payers in Europe should consider the co-financing of innovative high-risk devices used in premarket clinical trials, provided that these trials are well-controlled and have clinically relevant endpoints. This would reduce the financial hurdle for industry, which primarily comprises small and medium enterprises. This system already exists in the United States, where the Centers for Medicare and Medicaid Services (CMS) reimburse the cost of medical devices studied under the IDE if they meet certain criteria. The level of reimbursement is up to the cost of a currently marketed, similar product. As for medicines, specific regulations are needed for devices aimed at very small target populations.

\section{The Need for More Transparency in Europe}

In the United States, since 2007, full transparency is assured by the obligatory registration of trial protocols and study results, both for drugs and devices (11). The EU Clinical Trials Register now also provides protocol-related information to the public, but only for trials with medicines. The trial results for new medicines are made public in European Public Assessment Reports (EPARs). This is in sharp contrast with the approval system of devices in Europe (28) (Table 2).

There is a clear need for a public trial registry (25) and a publicly available summary describing the basis for granting a CE mark (4). Directive 2007/47/EC specifies that all parties involved in the application of directives are bound to observe confidentiality with regard to all information obtained in carrying out their tasks (27). The same Directive, however, also requires that clinical trials respect the Declaration of Helsinki. This suggests entering the trial in a publicly available trial registry before the first patient is entered. Negative and 
positive results should be published or otherwise made publicly available. This requirement of the Directive is clearly not respected in most cases.

The regulations in Europe allow for an early market introduction of high-risk medical devices at a time when the device cannot yet be considered to have an established indication in routine clinical use, suggesting that the use could be called experimental. Often, the confirmatory clinical development phase is still to be initiated or is ongoing.

European regulations of research ethics (e.g., the Declaration of Helsinki, The European Convention on Human Rights and Biomedicine, The Clinical Trials Directive) are mainly focused on clinical trials; however, there seems to be no reason to grant less protection to patients who are treated with a high-risk medical device in the experimental phase outside the context of a trial.

The new premarket procedure should result in an approved indication for the device and publicly available product documentation including the full results of all trials. This transparency is required to allow physicians to practice evidencebased medicine, patients to make an informed decision and HTA agencies to produce the correct assessment. It may, however, be appropriate to also study whether or not the rules that protect innovation need to be improved in view of this transparency requirement.

Transparency is also required in cases where the inventor of the device is also the principal investigator in a clinical trial of the device, a situation that is not unusual (32).

\section{Measures to be Taken at the Member State Level}

When awaiting a reworked Medical Device Directive, patient risk should be minimized at the Member State level by improving transparency with regard to the available clinical data and by limiting the market introduction of novel high-risk devices with minimal clinical data to centers with the necessary expertise. Preferably, this should be done under an appropriate research protocol (RCT if possible). This requires the commitment of the Competent Authorities, the marketing company, the physicians, and the hospitals.

The impact of HTA is limited as this occurs during the postmarket phase. Despite HTA reports concluding that evidence is insufficient for justifying reimbursement, marketing of the device continues and patients may be charged for its cost. Therefore, the ethical issues associated with the early market introduction of innovative high-risk devices should be studied further by the commissions and organizations that provide ethical guidance to physicians and hospitals.

With regard to patient safety of medical devices, the major control point in Europe is still postmarketing rather than premarketing surveillance, as is the case for medicines (18). Without active analysis, postmarket surveillance often remains an empty shell and a false reassurance of patient safety. It is hampered by an ongoing struggle with both under-reporting of the numerator (the number of adverse events) and a lack of data about the denominator (the total number of exposures) (21). These numbers are often lacking for devices (28). In this context, it has been reported that many manufacturers fail to fully fulfill their legal responsibility to collect product data once their device is on the market (4). Therefore, the industry statement that no information is available to suggest that patient safety in Europe has been compromised needs to be interpreted with caution (4).

\section{CONCLUSIONS}

HTA agencies in Europe are often confronted with a lack of high-quality clinical evidence when evaluating novel high-risk devices. We showed that this is linked to the European regulatory system that allows early market introduction based on limited clinical data. We recommend that Europe should require high-quality randomized trials demonstrating clinical efficacy and safety before granting the marketing approval of innovative high-risk medical devices. To allow physicians to practice evidence-based medicine, patients to make an informed decision and HTA agencies to produce the correct assessment, a major improvement in the transparency of information is also needed for such devices in Europe.

\section{CONTACT INFORMATION}

Frank Hulstaert, MD, MSc, Senior Expert, Belgian Health Care Knowledge Centre (KCE), Brussels, Belgium; Mattias Neyt, MSc, PhD, Health Economist, KCE; Imgard Vinck, MSc, Lawyer, KCE; Sabine Stordeur, RN, MA, PhD, Senior Expert in Clinical and Health Services Research, KCE; Mirjana Huić, MD, MSc, Assistant Director and Head of Department for Development, Research and Health Technology Assessment, Agency for Quality and Accreditation in Health Care and Social Welfare, Zagreb, Croatia; Stefan Sauerland, MD, MPH, Head of Department Non-Drug Interventions, Institute for Quality and Efficiency in Healthcare (IQWiG), Cologne, Germany; Marja R. Kuijpers, MSc, PharmD, Advisor, Dutch Health Care Insurance Board (CVZ), The Netherlands Payam Abrishami, MD, MA, Health Services Researcher, CVZ; Hindrik Vondeling, PhD, Associate Professor in Health Technology Assessment (HTA), Centre for Applied Health Services Research and Technology Assessment (CAST), University of Southern Denmark, Odense, Denmark; Bruno Flamion, MD, PhD, Professor in Physiology and Pharmacology, University of Namur, Belgium; Silvio Garattini, MD, Professor of Chemotherapy and Pharmacology, Director, Mario Negri Institute for Pharmacological Research, Milano, Italy; Mira Pavlovic, MD, Deputy Director, Health Technology Division, Direction de l'Évaluation Médicale, Économique et de Santé Publique (DEMESP), Haute Autorité de Santé (HAS), Paris, France; Hans Van Brabandt, $\mathrm{MD}$, Cardiologist, KCE. 


\section{CONFLICTS OF INTEREST}

Stefan Sauerland has received honoraria from Ethicon EndoSurgery (Europe). The other authors report they have no potential conflicts of interest.

\section{REFERENCES}

1. Audry A, Ghislain J. Le Dispositif Medical. Paris: Presses Universitaires de France. 2009;55:57.

2. Challoner DR, Vodra WW. Medical devices and health-creating a new regulatory framework for moderate-risk devices. $N$ Engl J Med. 2011;365:977-979.

3. Chimowitz MI, Lynn MJ, Derdeyn CP, et al. Stenting versus aggressive medical therapy for intracranial arterial stenosis. $N$ Engl J Med. 2011;365:993-1003.

4. Cohen D, Billingsley M. Europeans are left to their own devices. BMJ. 2011;342:d2748.

5. Council of the European Union Council. Conclusions on innovation in the medical device sector. Luxembourg. http://www.consilium.europa.eu/ uedocs/cms_data/docs/pressdata/en/lsa/122397.pdf (accessed June 6, 2011).

6. Dhruva SS, Bero LA, Redberg RF. Strength of study evidence examined by the FDA in premarket approval of cardiovascular devices. JAMA. 2009;302:2679-2685.

7. European Commission MEDDEV 2.7/4 December 2010. Guidelines on medical devices. Clinical evaluation: A Guide for manufacturers and notified bodies. http://ec.europa.eu/consumers/sectors/medicaldevices/files/meddev/2_7_4_en.pdf.

8. European Commission. Commision decision of 19 April 2010 on the European Databank on Medical Devices (Eudamed) (2010/227/EU). Official Journal of the European Union. L 102/45, 23.4.2010; 2010.

9. Fann JI, Chronos N, Rowe SJ, et al. Evolving strategies for the treatment of valvular heart disease: Preclinical and clinical pathways for percutaneous aortic valve replacement. Catheter Cardiovasc Interv. 2008;71:434-440.

10. FDA Meeting Materials of the Anesthesiology and Respiratory Therapy Devices Panel. http://www.fda.gov/AdvisoryCommittees/Committees MeetingMaterials/MedicalDevices/MedicalDevicesAdvisoryCommittee/ AnesthesiologyandRespiratoryTherapyDevicesPanel/ucm 123785.htm (accessed January 11, 2012).

11. FDA. Food and Drug Administration Amendments Act of 2007. http:// frwebgate.access.gpo.gov/cgi-bin/getdoc.cgi?dbname=110_cong_public _laws\&docid=f:pub1085.110 (accessed January 11, 2012).

12. Fraser AG, Krucoff MW, Brindis RG, Komajda M, Smith SC Jr. Commentary: International collaboration needed on device clinical standards. BMJ. 2011;342:d2952.

13. Fraser AG, Daubert JC, Van de Werf F, et al. Clinical evaluation of cardiovascular devices: Principles, problems, and proposals for European regulatory reform. Report of a policy conference of the European Society of Cardiology. Eur Heart J. 2011;32:1673-1686.

14. Freemantle N. Commentary: Evaluating and regulating device therapy. BMJ. 2011;342:d2839.

15. Heneghan C, Thompson M, Billingsley M, Cohen D. Medical-device recalls in the UK and the device-regulation process: Retrospective review of safety notices and alerts. BMJ Open. 2011;1:e000155.
16. Hines JZ, Lurie P, Yu E, Wolfe S. Left to their own devices: Breakdowns in United States medical device premarket review. PLoS Med. 2010;7:e1000280.

17. International Conference on Harmonisation. Guideline E8. General Considerations for Clinical Trials. Silver Spring, MD: US Food and Drug Administration; 1997.

18. Jefferys DB. The regulation of medical devices and the role of the Medical Devices Agency. Br J Clin Pharmacol. 2001;52:229-235.

19. Kaplan AV, Baim DS, Smith JJ, et al. Medical device development: From prototype to regulatory approval. Circulation. 2004;109:3068-3072.

20. Kramer DB, Mallis E, Zuckerman BD, Zimmerman BA, Maisel WH. Premarket clinical evaluation of novel cardiovascular devices: Quality analysis of premarket clinical studies submitted to the Food and Drug Administration 2000-2007. Am J Ther. 2010;17:2-7.

21. Lenzer J. Watching over the medical device industry. $B M J$. 2009;338:b2321.

22. Migliore A, Ratti M, Cerbo M, Jefferson T. Health technology assessment: Managing the introduction and use of medical devices in clinical practice in Italy. Expert Rev Med Devices. 2009;6:251-257.

23. Roszek B, de Bruijn A, Pot J, van Drongelen A. Assessment of technical documentation of Class III medical devices. National Institute for Public Health and the Environment, The Netherlands (RIVM) Report 360050021/2010. 2010.

24. Saviola J. The FDA's role in medical device clinical studies of human subjects. J Neural Eng. 2005;2:S1-S4.

25. Sim I. Trial registration for public trust: Making the case for medical devices. J Gen Intern Med. 2008;23(Suppl 1):64-68.

26. Stordeur S, Gerkens S, Roberfroid D. Interspinous implants and pedicle screws for dynamic stabilization of lumbar spine: A rapid Health Technology Assessment. Vol. 116. Brussels: Belgian Health Care Knowledge Centre, KCE; 2009.

27. The Council of the European Communities. Directive 2007/47/EC of the European Parliament and of the Council of 5 September 2007 amending Council Directive 90/385/EEC on the approximation of the laws of the Member States relating to active implantable medical devices, Council Directive 93/42/EEC concerning medical devices and Directive 98/8/EC concerning the placing of biocidal products on the market. Brussels: Council of the European Communities; 2007.

28. Thompson M, Heneghan C, Billingsley M, Cohen D. Medical device recalls and transparency in the UK. BMJ. 2011;342:d2973.

29. Tinkler J. Regional differences in medical device. Applied development applied clinical trials. 2009 (February 3, 2009).

30. Van Brabandt H, Neyt M. Percutaneous heart valve implantation in congenital and degenerative valve disease. A rapid health technology assessment. Vol. 95. Brussels: Belgian Health Care Knowledge Centre, KCE; 2008.

31. Van Brabandt H, Neyt M. Endobronchial valves in the treatment of severe pulmonary emphysema: A rapid health technology assessment. Vol. 114. Brussels: Belgian Health Care Knowledge Centre, KCE; 2009.

32. Wilmshurst $\mathrm{P}$. The regulation of medical devices. BMJ. 2011;342:d2822.

33. World Health Organisation. Clinical evidence for medical devices: Regulatory processes focussing on Europe and the United States of America. Background paper 3. Medical devices: Managing the mismatch. An outcome of the Priority Medical Devices project. Geneva: WHO; 2010. 